\title{
Chromopolarizability of Charmonium and $\pi \pi$ Final State Interaction Revisited
}

\author{
Yun-Hua Chen (1D \\ School of Mathematics and Physics, University of Science and Technology Beijing, Beijing 100083, China \\ Correspondence should be addressed to Yun-Hua Chen; yhchen@ustb.edu.cn
}

Received 16 January 2019; Accepted 7 March 2019; Published 23 April 2019

Guest Editor: Tao Luo

Copyright ( 2019 Yun-Hua Chen. This is an open access article distributed under the Creative Commons Attribution License, which permits unrestricted use, distribution, and reproduction in any medium, provided the original work is properly cited. The publication of this article was funded by $\mathrm{SCOAP}^{3}$.

\begin{abstract}
The chromopolarizability of a quarkonium describes the quarkonium's interaction with soft gluonic fields and can be measured in the heavy quarkonium decay. Within the framework of dispersion theory which considers the $\pi \pi$ final state interaction (FSI) model-independently, we analyze the transition $\psi^{\prime} \longrightarrow J / \psi \pi^{+} \pi^{-}$and obtain the chromopolarizability $\alpha_{\psi^{\prime} \psi}$ and the parameter $\kappa$. It is found that the $\pi \pi$ FSI plays an important role in extracting the chromopolarizability from the experimental data. The obtained chromopolarizability with the FSI is reduced to about $1 / 2$ of that without the FSI. With the FSI, we determine the chromopolarizability $\left|\alpha_{\psi^{\prime} \psi}\right|=(1.44 \pm 0.02) \mathrm{GeV}^{-3}$ and the parameter $\kappa=0.139 \pm 0.005$. Our results could be useful in studying the interactions of charmonium with light hadrons.
\end{abstract}

\section{Introduction}

The chromopolarizability $\alpha$ of a quarkonium parametrizes the quarkonium's effective interaction with soft gluons, and it is an important quantity in the heavy quark effective theory. Within the multipole expansion in QCD in terms of the chromopolarizability, many processes can be described, including the hadronic transitions between quarkonium resonances $[1,2]$ and the interaction of slow quarkonium with a nuclear medium [3]. A recent interest of the chromopolarizabilities of $J / \psi$ and $\psi^{\prime}$ comes from the hadrocharmonium [3-8] interpretation of the $P_{c}^{+}(4380)$ and $P_{c}^{+}(4450)$ observed by the LHCb Collaboration, and it is found that the $P_{c}^{+}(4450)$ can be interpreted as a $\psi^{\prime}$-nucleon bound state if $\alpha_{\psi^{\prime}} / \alpha_{J / \psi} \simeq 15$ [9].

There are a few studies of the chromopolarizabilities of $J / \psi$ and $\psi^{\prime}$, some of which are not in line with each other. Calculated in the large- $N_{c}$ limit in the heavy quark approximation, the values of the chromopolarizabilities of the $J / \psi$ and $\psi^{\prime}$ are obtained: $\alpha_{J / \psi} \approx 0.2 \mathrm{GeV}^{-3}$ and $\alpha_{\psi^{\prime}} \approx 12 \mathrm{GeV}^{-3}[6,7,10,11]$. Within a quarkoniumnucleon effective field theory, the chromopolarizability of the $J / \psi$ is determined through fitting the lattice QCD data [12] of the $J / \psi$-nucleon potential, and the result is $\alpha_{J / \psi}=$ $0.24 \mathrm{GeV}^{-3}[13,14]$. Based on an effective potential formalism given in [15] and a recent lattice QCD calculation [16], the chromopolarizabilities of $J / \psi$ are extracted to be $\alpha_{J / \psi}=$ $(1.6 \pm 0.8) \mathrm{GeV}^{-3}$ [9]. On the other hand, the determination of the transitional chromopolarizability $\alpha_{\psi^{\prime} \psi} \equiv \alpha_{\psi^{\prime} \rightarrow J / \psi}$ is of importance since it acts a reference benchmark for either of the diagonal terms due to the Schwartz inequality: $\alpha_{J / \psi} \alpha_{\psi^{\prime}} \geq$ $\alpha_{\psi^{\prime} \psi}^{2}$ [4]. The perturbative prediction in the large $N_{c}$ limit is $\alpha_{\psi^{\prime} \psi} \approx-0.6 \mathrm{GeV}^{-3}[6,7,10,11]$. While being extracted from the process of $\psi^{\prime} \longrightarrow J / \psi \pi \pi$, the result is $\left|\alpha_{\psi^{\prime} \psi}\right| \approx 2 \mathrm{GeV}^{-3}$ $[4,17]$. Taking account of the $\pi \pi$ FSI in a chiral unitary approach, it is found that the value of $\left|\alpha_{\psi^{\prime} \psi}\right|$ may be reduced to about $1 / 3$ of that without the $\pi \pi$ FSI [18].

Since the FSI plays an important role in the heavy quarkonium transitions and modifies the value of $\alpha_{\psi^{\prime} \psi}$ significantly, it is thus necessary to account for the FSI properly. In this work we will use the dispersion theory to take into account of the $\pi \pi$ FSI and extract the value of $\alpha_{\psi^{\prime} \psi}$. Instead of the chiral unitary approach $[18,19]$, in which the scalar mesons $\left(\sigma, f_{0}(980)\right.$, and $\left.a_{0}(980)\right)$ are dynamically generated, in the dispersion theory the $\pi \pi$ FSI is treated in a modelindependent way consistent with $\pi \pi$ scattering data. Another update of our calculation is that we consider the FSIs of separate partial waves, namely, the $S$ - and $D$-waves, instead 
of only accounting for the $S$-wave as in the parametrization in $[17,18]$.

The theoretical framework is described in detail in Section 2. In Section 3, we fit the decay amplitudes to the data for the $\psi^{\prime} \longrightarrow J / \psi \pi^{+} \pi^{-}$transition and determine the chromopolarizability $\alpha_{\psi^{\prime} \psi}$ and the parameter $\kappa$. A brief summary will be presented in Section 4.

\section{Theoretical Framework}

First we define the Mandelstam variables of the decay process $\psi^{\prime}\left(p_{a}\right) \longrightarrow J / \psi\left(p_{b}\right) \pi\left(p_{c}\right) \pi\left(p_{d}\right)$

$$
\begin{aligned}
& s=\left(p_{c}+p_{d}\right)^{2}, \\
& t=\left(p_{a}-p_{c}\right)^{2}, \\
& u=\left(p_{a}-p_{d}\right)^{2} .
\end{aligned}
$$

The amplitude for the $\pi^{+} \pi^{-}$transition between $S$-wave states $A$ and $B$ of heavy quarkonium can be written as $[4,13]$

$$
\begin{aligned}
M_{A B} & =2 \sqrt{m_{A} m_{B}} \alpha_{A B}\left\langle\pi^{+}\left(p_{c}\right) \pi^{-}\left(p_{d}\right)\left|\frac{1}{2} \mathbf{E}^{a} \cdot \mathbf{E}^{a}\right| 0\right\rangle \\
& =\frac{8 \pi^{2}}{b} \sqrt{m_{A} m_{B}} \alpha_{A B}\left(\kappa_{1} p_{c}^{0} p_{d}^{0}-\kappa_{2} p_{c}^{i} p_{d}^{i}\right),
\end{aligned}
$$

where the factor $2 \sqrt{m_{A} m_{B}}$ appears due to the relativistic normalization of the decay amplitude, $\alpha_{A B}$ is the chromopolarizability, and $\mathbf{E}^{a}$ denotes the chromoelectric field. $b$ is the first coefficient of the QCD beta function, $b=(11 / 3) N_{c}-$ $(2 / 3) N_{f}$, where $N_{c}=3$ and $N_{f}=3$ are the number of colors and of light flavors, respectively. $\kappa_{1}=2-9 \kappa / 2$, and $\kappa_{2}=2+3 \kappa / 2$, where $\kappa$ is a parameter that can be determined from the data.

The above result of the QCD multipole expansion together with the soft-pion theorem can be reproduced by constructing a chiral effective Lagrangian for the $\psi^{\prime} \longrightarrow$ $J / \psi \pi \pi$ transition. Since the spin-dependent interactions are suppressed by the charm mass, the heavy quarkonia can be expressed in terms of spin multiplets, and one has $J \equiv \psi$. $\boldsymbol{\sigma}+\eta_{c}$, where $\boldsymbol{\sigma}$ contains the Pauli matrices and $\psi$ and $\eta_{c}$ annihilates the $\psi$ and $\eta_{c}$ states, respectively [20]. The effective Lagrangian, at the leading order in the chiral as well as the heavy quark nonrelativistic expansion, reads [21-23]

$$
\begin{aligned}
\mathscr{L}_{\psi \psi^{\prime} \pi \pi}= & \frac{c_{1}}{2}\left\langle J^{\dagger} J^{\prime}\right\rangle\left\langle u_{\mu} u^{\mu}\right\rangle+\frac{c_{2}}{2}\left\langle J^{\dagger} J^{\prime}\right\rangle\left\langle u_{\mu} u_{\nu}\right\rangle v^{\mu} v^{v} \\
& + \text { h.c. }
\end{aligned}
$$

where $v^{\mu}=(1,0)$ is the velocity of the heavy quark. The Goldstone bosons of the spontaneous breaking of chiral symmetry can be parametrized according to

$$
\begin{aligned}
& u_{\mu}=i\left(u^{\dagger} \partial_{\mu} u-u \partial_{\mu} u^{\dagger}\right) \\
& u^{2}=e^{i \Phi / F_{\pi}}, \quad \Phi=\left(\begin{array}{cc}
\pi^{0} & \sqrt{2} \pi^{+} \\
\sqrt{2} \pi^{-} & -\pi^{0}
\end{array}\right),
\end{aligned}
$$

where $F_{\pi}=92.2 \mathrm{MeV}$ denotes the pion decay constant.
The amplitude obtained by using the effective Lagrangians in (3) is

$$
M(s, t, u)=-\frac{4}{F_{\pi}^{2}}\left(c_{1} p_{c} \cdot p_{d}+c_{2} p_{c}^{0} p_{d}^{0}\right) .
$$

Matching the amplitude in (2) to that in (5), we can express the low-energy couplings in the chiral effective Lagrangian in terms of the chromopolarizability $\alpha_{A B}$ and the parameter $\kappa$

$$
\begin{aligned}
& c_{1}=-\frac{\pi^{2} \sqrt{m_{\psi^{\prime}} m_{\psi}} F_{\pi}^{2}}{b} \alpha_{\psi^{\prime} \psi}(4+3 \kappa), \\
& c_{2}=\frac{12 \pi^{2} \sqrt{m_{\psi^{\prime}} m_{\psi}} F_{\pi}^{2}}{b} \alpha_{\psi^{\prime} \psi} \kappa .
\end{aligned}
$$

The partial-wave decomposition of $M(s, t, u)$ can be easily performed by using the relation

$$
p_{c}^{0} p_{d}^{0}=\frac{1}{4}\left(s+\mathbf{q}^{2}\right)-\frac{1}{4} \mathbf{q}^{2} \sigma_{\pi}^{2} \cos ^{2} \theta
$$

where $\mathbf{q}$ is the 3-momentum of the final vector meson in the rest frame of the initial state with $|\mathbf{q}|=\left\{\left[\left(m_{\psi^{\prime}}+m_{\psi}\right)^{2}-\right.\right.$ $\left.s] /\left[\left(m_{\psi^{\prime}}-m_{\psi}\right)^{2}-s\right]\right\}^{1 / 2} /\left(2 m_{\psi^{\prime}}\right), \sigma_{\pi} \equiv \sqrt{1-4 m_{\pi}^{2} / s}$, and $\theta$ is the angle between the 3-momentum of the $\pi^{+}$in the rest frame of the $\pi \pi$ system and that of the $\pi \pi$ system in the rest frame of the initial $\psi^{\prime}$.

Parity and $C$-parity conservations require the pion pair to have even relative angular momentum $l$. We only consider the $S$ - and $D$-wave components in this study, neglecting the effects of higher partial waves. Explicitly, the $S$ - and $D$-wave components of the amplitude read

$$
\begin{aligned}
& M_{0}^{\chi}(s) \\
& \quad=-\frac{2}{F_{\pi}^{2}}\left\{c_{1}\left(s-2 m_{\pi}^{2}\right)+\frac{c_{2}}{2}\left[s+\mathbf{q}^{2}\left(1-\frac{\sigma_{\pi}^{2}}{3}\right)\right]\right\}, \\
& M_{2}^{\chi}(s)=\frac{2}{3 F_{\pi}^{2}} c_{2} \mathbf{q}^{2} \sigma_{\pi}^{2} .
\end{aligned}
$$

There are strong FSI in the $\pi \pi$ system especially in the isospin-0 $S$-wave, which can be taken into account modelindependently using dispersion theory [22-31]. We will use the Omnès solution to obtain the amplitude including FSI. In the region of elastic $\pi \pi$ rescattering, the partial-wave unitarity conditions read

$$
\operatorname{Im} M_{l}(s)=M_{l}(s) \sin \delta_{l}^{0}(s) e^{-i \delta_{l}^{0}(s)}
$$

Below the inelastic threshold, the phases $\delta_{l}^{I}$ of the partialwave amplitudes of isospin $I$ and angular momentum $l$ coincide with the $\pi \pi$ elastic phase shifts modulo $n \pi$, as required by Watson's theorem $[32,33]$. It is known that the standard Omnès solution of (9) is as follows:

$$
M_{l}(s)=P_{l}^{n}(s) \Omega_{l}^{0}(s),
$$



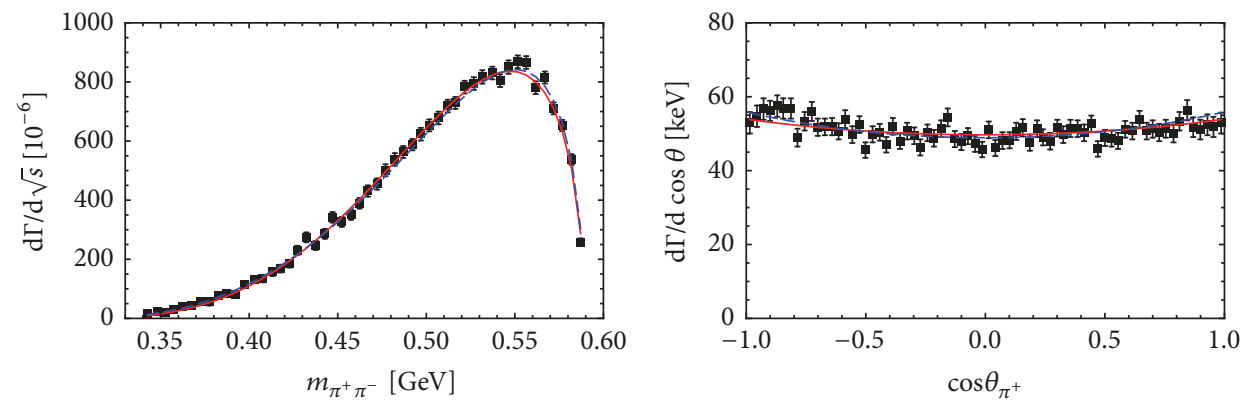

Figure 1: Simultaneous fit to the $\pi \pi$ invariant mass distributions and the helicity angle distributions in $\psi^{\prime} \longrightarrow J / \psi \pi^{+} \pi^{-}$. The red solid and blue dashed curves represent the theoretical fit results with $\pi \pi$ FSI and without $\pi \pi$ FSI cases, respectively. The data are taken from [37].

where the $P_{l}^{n}(s)$ is a polynomial, and the Omnès function is defined as [34]

$$
\Omega_{l}^{I}(s)=\exp \left\{\frac{s}{\pi} \int_{4 m_{\pi}^{2}}^{\infty} \frac{\mathrm{d} x}{x} \frac{\delta_{l}^{I}(x)}{x-s}\right\} .
$$

At low energies, $M_{0}(s)$ and $M_{2}(s)$ can be matched to the chiral representation. Namely, in the limit of switching off the $\pi \pi$ FSI, i.e., $\Omega_{l}^{0}(s) \equiv 1$, the polynomials $P_{l}^{n}(s)$ can be identified exactly with the expressions given in (8). Therefore, the amplitudes including the FSI take the form

$$
\begin{aligned}
& M_{0}(s) \\
& =-\frac{2}{F_{\pi}^{2}}\left[c_{1}\left(s-2 m_{\pi}^{2}\right)+\frac{c_{2}}{2}\left(s+\mathbf{q}^{2}\left(1-\frac{\sigma_{\pi}^{2}}{3}\right)\right)\right] \\
& \cdot \Omega_{0}^{0}(s), \\
& M_{2}(s)=\frac{2}{3 F_{\pi}^{2}} c_{2} \mathbf{q}^{2} \sigma_{\pi}^{2} \Omega_{2}^{0}(s) .
\end{aligned}
$$

Now we discuss the $\pi \pi$ phase shifts used in the calculation of the Omnès functions. For the $S$-wave, we use the phase of the nonstrange pion scalar form factor as determined in [35], which yields a good description below the onset of the $K \bar{K}$ threshold. For the $D$-wave, we employ the parametrization for $\delta_{2}^{0}$ given by the Madrid-Kraków collaboration [36]. Both phases are guided smoothly to $\pi$ for $s \longrightarrow \infty$.

It is then straightforward to calculate the $\pi \pi$ invariant mass spectrum and helicity angular distribution for $\psi^{\prime} \longrightarrow$ $J / \psi \pi^{+} \pi^{-}$using

$$
\begin{aligned}
& \frac{\mathrm{d} \Gamma}{\mathrm{d} \sqrt{s} \mathrm{~d} \cos \theta} \\
& \quad=\frac{\sqrt{s} \sigma_{\pi}|\mathbf{q}|}{128 \pi^{3} m_{\psi^{\prime}}^{2}}\left|M_{0}(s)+M_{2}(s) P_{2}(\cos \theta)\right|^{2},
\end{aligned}
$$

where the Legendre polynomial $P_{2}(\cos \theta)=\left(3 \cos ^{2} \theta-1\right) / 2$.

\section{Phenomenological Discussion}

The unknown parameters are the low-energy constants $c_{1}$ and $c_{2}$ in the chiral Lagrangian (3), which can be expressed in
TABLE 1: The parameter results from the fits of the $\psi^{\prime} \longrightarrow \psi \pi \pi$ processes with and without the $\pi \pi$ FSI.

\begin{tabular}{lcc}
\hline & Without $\pi \pi$ FSI & With $\pi \pi$ FSI \\
\hline$\left|\alpha_{\psi^{\prime} \psi}\right|\left(\mathrm{GeV}^{-3}\right)$ & $2.37 \pm 0.02$ & $1.44 \pm 0.02$ \\
$\kappa$ & $0.135 \pm 0.005$ & $0.139 \pm 0.005$ \\
\hline$\frac{\chi^{2}}{\text { d.o.f }}$ & $\frac{115.3}{120-2}=0.98$ & $\frac{117.6}{120-2}=1.00$ \\
\hline
\end{tabular}

terms of the chromopolarizability $\alpha_{\psi^{\prime} \psi}$ and the parameter $\kappa$ as in (6). In order to determine $\alpha_{\psi^{\prime} \psi}$ and $\kappa$, we fit the theoretical results to the experimental $\pi^{+} \pi^{-}$invariant mass spectra and the helicity angular distribution from the BES $\psi^{\prime} \longrightarrow J / \psi \pi^{+} \pi^{-}$decay data [37] and the corresponding decay width $\Gamma\left(\psi^{\prime} \longrightarrow J / \psi \pi^{+} \pi^{-}\right)$[38]. The fit results are plotted in Figure 1, where the red solid and blue dashed curves represent the results with or without the $\pi \pi$ FSI, respectively. The fit parameters as well as the $\chi^{2} /$ d.o.f. are shown in Table 1 . One observes that the experimental data can be well described regardless of whether the FSI is included. This is due to the simple shapes of the $\pi \pi$ invariant mass distribution and the helicity angular distribution in this process and does not mean the FSI is not important. Since the dipion mass invariant mass reaches about $600 \mathrm{MeV}$ in such a decay, the $\pi \pi$ FSI is known to be strong in this energy range and needs to be considered. On the other hand, one can readily see from (6) and (8) that while the chromopolarizability $\alpha_{\psi^{\prime} \psi}$ determines the overall decay rate, the parameter $\kappa$ characterizes the $D$ wave contribution, and we do not find significant correlation between $\alpha_{\psi^{\prime} \psi}$ and $\kappa$.

We observe that the $\pi \pi$ FSI modifies the value of the chromopolarizability $\alpha_{\psi^{\prime} \psi}$ significantly, and resultant value with the FSI is almost $1 / 2$ of that without the FSI. The obtained value with the FSI, $\left|\alpha_{\psi^{\prime} \psi}\right|=(1.44 \pm 0.02) \mathrm{GeV}^{-3}$, coincides with the suspicion $\alpha_{J / \psi} \geq\left|\alpha_{\psi^{\prime} \psi}\right|$ [3] with the value $\alpha_{J / \psi}=$ (1.6 \pm 0.8$) \mathrm{GeV}^{-3}$ from the calculation [9] based on the recent lattice QCD data of $J / \psi$-nucleon potential [16]. It should be mentioned that the value of $\alpha_{\psi^{\prime} \psi}$ with the FSI obtained here is different from the one in [18], $\left|\alpha_{\psi^{\prime} \psi}\right|=(0.83 \pm 0.01) \mathrm{GeV}^{-3}$, and also our result without the FSI slightly differs from those in $[17,18]$. The reasons are that the chiral unitary approach instead of dispersion theory is used to account for the FSI 
in [18], and we use the updated experimental data $[37,38]$ and a general theoretical amplitude rather than the one only containing the $S$-wave as employed in $[17,18]$.

For the parameter $\kappa$, as shown in Table 1 its value is affected little by the $\pi \pi$ FSI. One notes that a detailed study of the $\psi^{\prime} \longrightarrow J / \psi \pi^{+} \pi^{-}$process using the Novikov-Shifman model [2] has been performed by BES [39], and based on the joint $m_{\pi^{+} \pi^{-}} \cos \theta_{\pi^{+}}$distribution this parameter was determined as $\kappa=0.183 \pm 0.002 \pm 0.003$. We have tried fitting the same old BES data [39] and our $\kappa$ changes slightly and is still much smaller than the BES one. In the Novikov-Shifman model, the $\psi^{\prime} \longrightarrow J / \psi \pi^{+} \pi^{-}$amplitude reads [2]

$$
\begin{aligned}
M & \propto\left\{s-\kappa\left(m_{\psi^{\prime}}-m_{\psi}\right)^{2}\left(1+\frac{2 m_{\pi}^{2}}{s}\right)\right. \\
& \left.+\frac{3}{2} \kappa\left[\left(m_{\psi^{\prime}}-m_{\psi}\right)^{2}-s\right] \sigma_{\pi}^{2}\left(\cos ^{2} \theta-\frac{1}{3}\right)\right\} .
\end{aligned}
$$

If we make the same approximation, namely, neglect the $O\left(m_{\pi}^{2}\right)$ terms except the $m_{\pi}^{2} / s$ ones, as in [2] and set $\left(m_{\psi^{\prime}}+\right.$ $\left.m_{\psi}\right)^{2}-s \approx\left(m_{\psi^{\prime}}+m_{\psi}\right)^{2}$ in the expression of 3-momentum $\mathbf{q}$, our amplitude without the $\pi \pi$ FSI agrees with (15). While numerically we find that some neglected $O\left(m_{\pi}^{2}\right)$ terms are at the same order as the $\kappa\left(m_{\psi^{\prime}}-m_{\psi}\right)^{2}$ term in (15), this may account for the difference of $\kappa$ between ours and that in [39]. On the other hand, we have checked that the contribution of the $D$-wave, which is characterized by the parameter $\kappa$, to the total rate is less than two percent, and the same observation has been made in [39].

\section{Conclusions}

We have used dispersion theory to study the $\pi \pi$ FSI in the decay $\psi^{\prime} \longrightarrow J / \psi \pi^{+} \pi^{-}$. Through fitting the data of the $\pi \pi$ mass spectra and the angular $\cos \theta$ distributions, the values of the chromopolarizability $\alpha_{\psi^{\prime} \psi}$ and the parameter $\kappa$ are determined. It is found that the effect of the $\pi \pi$ FSI is quite sizeable in the chromopolarizability $\alpha_{\psi^{\prime} \psi}$, and the one with FSI is almost $1 / 2$ of that without FSI. The parameter $\kappa$, which accounts for the $D$-wave contribution, is affected little by the $\pi \pi$ FSI. The results obtained in this work would be valuable to understand the chromopolarizability of charmonia and will have applications for the studies of the nucleon-charmonia interaction.

\section{Data Availability}

All the data used in this work are from $[37,38]$.

\section{Conflicts of Interest}

The author declares that they have no conflicts of interest.

\section{Acknowledgments}

We acknowledge Feng-Kun Guo for the proposal for this work and for the useful comments on the manuscript. This research is supported in part by the Fundamental Research Funds for the Central Universities under Grant no. 06500077.

\section{References}

[1] M. Voloshin and V. Zakharov, "Measuring quantum-chromodynamic anomalies in hadronic transitions between quarkonium states," Physical Review Letters, vol. 45, no. 9, pp. 688-691, 1980.

[2] V. A. Novikov and M. A. Shifman, "Comment on the $\psi^{\prime} \longrightarrow \mathrm{J} / \psi \pi \pi$ decay," Zeitschrift für Physik C Particles and Fields, vol. 8, no. 1, pp. 43-47, 1981.

[3] A. Sibirtsev and M. B. Voloshin, "Interaction of slow $J / \psi$ and $\psi^{\prime}$ with nucleons," Physical Review D, vol. 71, Article ID 076005, 2005.

[4] M. B. Voloshin, "Charmonium," Progress in Particle and Nuclear Physics, vol. 61, no. 455, 2008.

[5] S. Dubynskiy and M. Voloshin, "Hadro-charmonium," Physics Letters B, vol. 666, no. 4, pp. 344-346, 2008.

[6] M. I. Eides, V. Y. Petrov, and M. V. Polyakov, "Narrow nucleon$\psi(2 S)$ bound state and LHCb pentaquarks," Physical Review D: Particles, Fields, Gravitation and Cosmology, vol. 93, Article ID 054039, 2016.

[7] M. I. Eides, V. Y. Petrov, and M. V. Polyakov, "Pentaquarks with hidden charm as hadroquarkonia," The European Physical Journal C, vol. 78, no. 36, 2018.

[8] K. Tsushima, D. H. Lu, G. Krein, and A. W. Thomas, "J/Inuclear bound states," Physical Review C: Nuclear Physics, vol. 83, no. 6, Article ID 065208, 2011.

[9] M. V. Polyakov and P. Schweitzer, "Determination of $J / \psi$ chromoelectric polarizability from lattice data," Physical Review D, vol. 98, no. 3, Article ID 034030, pp. 1-7, 2018.

[10] M. E. Peskin, "Short-distance analysis for heavy-quark systems: (I). Diagrammatics," Nuclear Physics B, vol. 156, no. 3, pp. 365390, 1979.

[11] G. Bhanot and M. E. Peskin, "Short-distance analysis for heavyquark systems: (I). Diagrammatics,” Nuclear Physics B, vol. 156, no. 3, pp. 365-390, 1979.

[12] T. Kawanai and S. Sasaki, "Charmonium-nucleon potential from lattice QCD," Physical Review D, vol. 82, no. 9, Article ID 091501, 2010.

[13] N. Brambilla, G. Krein, J. Tarrús Castellà, and A. Vairo, "Longrange properties of $1 S$ bottomonium states," Physical Review D: Particles, Fields, Gravitation and Cosmology, vol. 93, Article ID 054002, 2016.

[14] J. Tarrs Castell and G. Krein, "Effective field theory for the nucleon-quarkonium interaction," Physical Review D, vol. 98, no. 1, Article ID 014029, 2018.

[15] M. B. Voloshin, "Precoulombic asymptotics for energy levels of heavy quarkonium," Soviet Journal of Nuclear Physics, vol. 36, no. 143, pp. 247-255, 1982.

[16] T. Sugiura, Y. Ikeda, and N. Ishii, "Charmonium-nucleon interactions from the time-dependent HAL QCD method," in Proceedings of the 35th International Symposium on Lattice Field Theory (Lattice 2017), vol. 175, pp. 1-8, 2018.

[17] M. B. Voloshin, "Quarkonium chromo-polarizability from the decays $\mathrm{J} / \psi(\Upsilon) \longrightarrow \pi \pi \ell^{+} \ell^{-}$," Modern Physics Letters $A$, vol. 19, no. 9, pp. 665-670, 2004.

[18] F. K. Guo, P. N. Shen, and H. C. Chiang, "Chromopolarizability and $\pi \pi$ final state interaction," Physical Review D, vol. 74, Article ID 014011, pp. 11-15, 2006. 
[19] J. A. Oller and E. Oset, "Chiral symmetry amplitudes in the $\mathrm{S}$-wave isoscalar and isovector channels and the $\sigma, \mathrm{f}_{0}(980)$, a0(980) scalar mesons," Nuclear Physics A, vol. 620, pp. 438-456, 1997.

[20] M. Cleven, F.-K. Guo, C. Hanhart, and U.-G. Meißner, "Bound state nature of the exotic $\mathrm{Z}_{b}$ states," The European Physical Journal $A$, vol. 47, no. 120, 2011.

[21] T. Mannel and R. Urech, "Hadronic decays of excited heavy quarkonia," Zeitschrift für Physik C Particles and Fields, vol. 73, no. $541,1997$.

[22] Y. Chen, J. T. Daub, F. Guo, B. Kubis, U. Meißner, and B. Zou, "Effect of $Z_{b}$ states on $\Upsilon(3 S) \longrightarrow \Upsilon(1 S) \pi \pi$ decays," Physical Review D, vol. 93, no. 3, Article ID 034030, 2016.

[23] Y. Chen, M. Cleven, J. T. Daub et al., "Effects of $Z_{b}$ states and bottom meson loops on $\Upsilon(4 S) \longrightarrow \Upsilon(1 S, 2 S) \pi^{+} \pi^{-}$transitions," Physical Review D, vol. 95, Article ID 034022, 2017.

[24] X. Kang, B. Kubis, C. Hanhart, and U. Meissner, " $B_{l 4}$ decays and the extraction of $\left|V_{u b}\right|$," Physical Review D, vol. 89, no. 5, Article ID 053015, 2014.

[25] B. Kubis and J. Plenter, "Anomalous decay and scattering processes of the $\eta$ meson," The European Physical Journal C, vol. 75, no. 283, 2015.

[26] T. Isken, B. Kubis, S. P. Schneider, and P. Stoffer, "Dispersion relations for $\eta$ ' $\longrightarrow \eta \pi \pi$," The European Physical Journal, vol. 77, p. 489, 2017.

[27] S. Ropertz, C. Hanhart, and B. Kubis, "A new parametrization for the scalar pion form factors," The European Physical Journal C, vol. 78, no. 12, article no. 1000, 2018.

[28] L.-Y. Dai and M. R. Pennington, "Two photon couplings of the lightest isoscalars from BELLE data," Physics Letters B, vol. 736, pp. 11-15, 2014.

[29] L.-Y. Dai and M. R. Pennington, "Comprehensive amplitude analysis of $\gamma \gamma \longrightarrow \pi^{+} \pi^{-}, \pi^{0} \pi^{0}$ and $\bar{K} K$ below $1.5 \mathrm{GeV}$," Physical Review D, vol. 90, Article ID 036004, 2014.

[30] L.-Y. Dai and M. R. Pennington, "Pion polarizabilities from a $\gamma \gamma \longrightarrow \pi \pi$ analysis," Physical Review D: Particles, Fields, Gravitation and Cosmology, vol. 94, no. 4, Article ID 116021, 2016.

[31] L.-Y. Dai and M. R. Pennington, "Pascalutsa-Vanderhaeghen light-by-light sum rule from photon-photon collisions," Physical Review D, vol. 97, Article ID 036012, 2018.

[32] K. M. Watson, "The effect of final state interactions on reaction cross sections," Physical Review, vol. 88, no. 1163, 1952.

[33] K. M. Watson, "Some general relations between the photoproduction and scattering of $\pi$ mesons," Physical Review A: Atomic, Molecular and Optical Physics, vol. 95, no. 228, 1954.

[34] R. Omnès, "On the solution of certain singular integral equations of quantum field theory," Nuovo Cimento, vol. 8, pp. 316$326,1958$.

[35] M. Hoferichter, C. Ditsche, B. Kubis, U.-G. Mei, and U.-G. Meißner, "Dispersive analysis of the scalar form factor of the nucleon," Journal of High Energy Physics, vol. 06, article no. 063, 2012.

[36] R. García-Martín, R. Kamiński, J. R. Peláez, J. Ruiz de Elvira, and F. J. Ynduráin, "Pion-pion scattering amplitude. IV: improved analysis with once subtracted roy-like equations up to 1100 MeV," Physical Review D, vol. 83, no. 7, Article ID 074004, 2011.

[37] M. Ablikim, J. Z. Bai, Y. Ban et al., "Production of $\sigma$ in $\psi(2 S) \longrightarrow$ $\pi^{+} \pi^{-} J / \psi$," Physics Letters B, vol. 645, no. 19, 2007.
[38] M. Tanabashi, U. Nagoya, K. M. I. Nagoya et al., "Review of Particle Physics," Physical Review D, vol. 98, no. 3, Article ID 030001, 2018.

[39] J. Z. Bai, Y. Ban, J. G. Bian et al., " $\psi(2 S) \longrightarrow \pi^{+} \pi^{-} J / \psi$ decay distributions," Physical Review D, vol. 62, Article ID 032002, 2000 . 

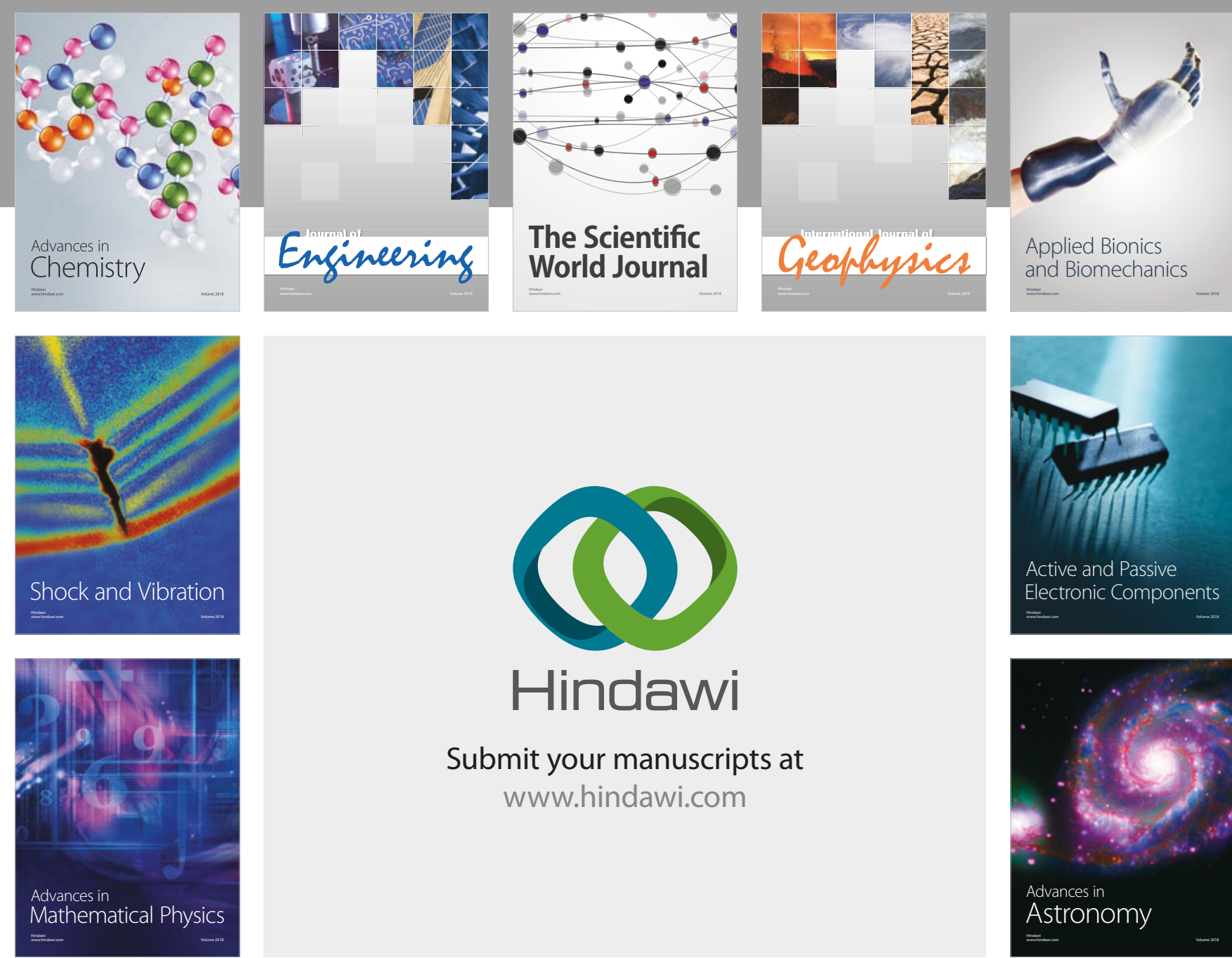

Submit your manuscripts at

www.hindawi.com

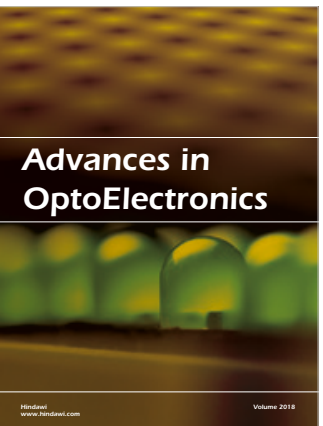

\section{Rotcting Machinery}
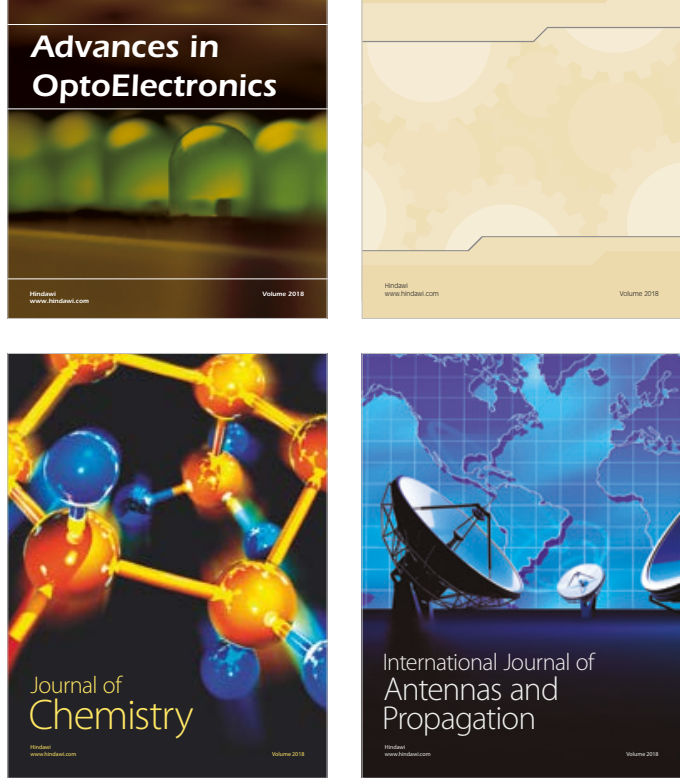

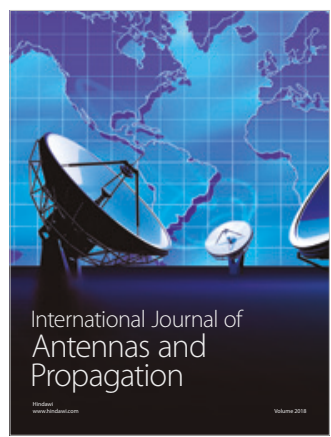

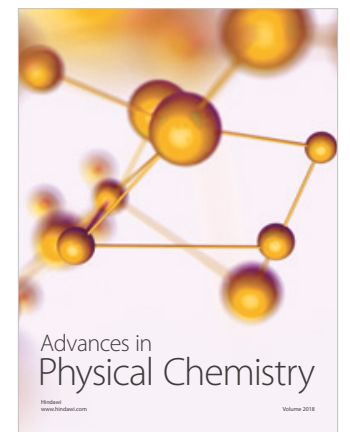

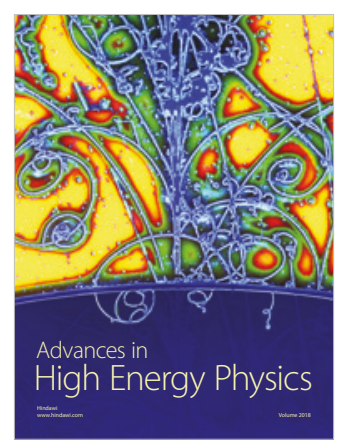

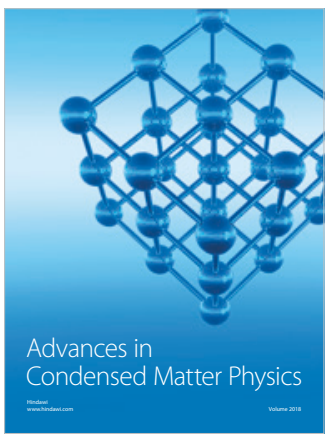

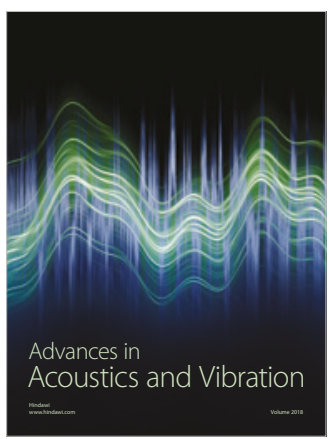

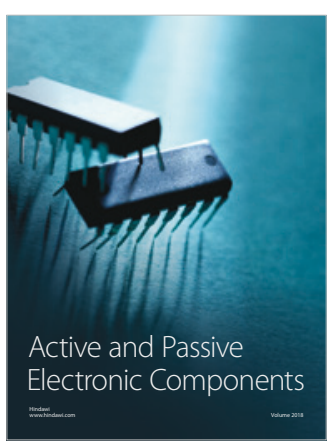
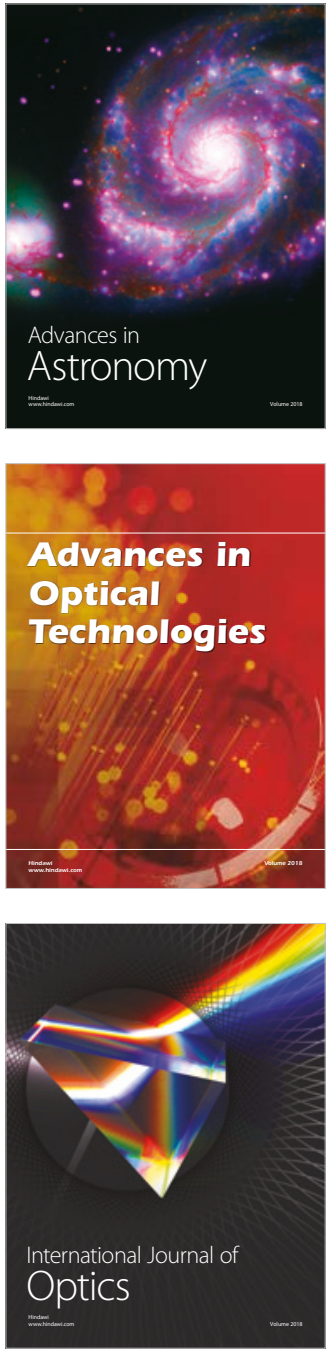